$<$ Back to results 1 of 1

Cited by 0 documents

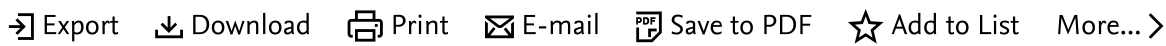

Full Tert

Neurotoxicity Research • Volume 39, Issue 6, Pages 1732 - 1746 • December 2021

Document type

Article

Source type

Journal

ISSN

10298428

DOI

10.1007/s12640-021-00417-y

Publisher

Springer

CODEN

NURRF

Original language

English

PubMed ID

34570348 ᄀ

View less $\wedge$

\section{Metformin Therapy Attenuates Pro-inflammatory Microglia by Inhibiting NF- $\kappa B$ in Cuprizone Demyelinating Mouse Model of Multiple Sclerosis}

Abdi M. ${ }^{a}$, Pasbakhsh P. ${ }^{a}$, Shabani M.b, Nekoonam S. ${ }^{a}$, Sadeghi A. ${ }^{c}$, Fathi F. ${ }^{d}$, Abouzaripour M. ${ }^{\mathrm{e}}$, Mohamed W. ${ }^{\mathrm{f} g}$, Zibara K. ${ }^{\mathrm{h}}$ 四, Kashani I.R. ${ }^{\mathrm{a}}, \quad$ Zendedel A. ${ }^{\mathrm{i}}$

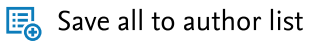

a Department of Anatomy, school of medicine, Tehran University of Medical Sciences, Tehran, Iran

${ }^{b}$ Department of Clinical Biochemistry, Tehran University of Medical Sciences, Tehran, Iran

${ }^{c}$ Department of Clinical Biochemistry, Faculty of medicine, Kerman University of Medical Sciences, Kerman, Iran

${ }^{d}$ Cellular and Molecular Research Center, Kurdistan University of Medical Sciences, Sanandaj, Iran

View additional affiliations $\vee$

Full text options
Inform me when this document is cited in Scopus:

Set citation alert >

Related documents

Metformin accelerates myelin recovery and ameliorates behavioral deficits in the animal model of multiple sclerosis via adjustment of AMPK/Nrf2/mTOR signaling and maintenance of endogenous oligodendrogenesis during brain self-repairing period

Sanadgol, N. , Barati, M. , Houshmand, F.

(2020) Pharmacological Reports

Investigation of Cuprizone Inactivation by Temperature

Heckers, S. , Held, N. ,

Kronenberg, J.

(2017) Neurotoxicity Research

Microglia polarization by methylprednizolone acetate accelerates cuprizone induced demyelination

Noorzehi, G. , Pasbakhsh, P. , Borhani-Haghighi, $M$.

(2018) Journal of Molecular Histology

View all related documents based on references

Find more related documents in Scopus based on:

Authors > Keywords > 
SciVal Topics

Metrics

Funding details

\section{Abstract}

Multiple sclerosis (MS) is a chronic disorder characterized by reactive gliosis, inflammation, and demyelination. Microglia plays a crucial role in the pathogenesis of MS and has the dynamic plasticity to polarize between pro-inflammatory (M1) and anti-inflammatory (M2) phenotypes. Metformin, a glucose-lowering drug, attenuates inflammatory responses by activating adenosine monophosphate protein kinase (AMPK) which suppresses nuclear factor kappa B (NF- $\mathrm{B}$ ). In this study, we indirectly investigated whether metformin therapy would regulate microglia activity in the cuprizone (CPZ)-induced demyelination mouse model of MS via measuring the markers associated with pro- and anti-inflammatory microglia . Evaluation of myelin by luxol fast blue staining revealed that metformin treatment $(\mathrm{CPZ}+\mathrm{Met})$ diminished demyelination, in comparison to $\mathrm{CPZ}$ mice. In addition, metformin therapy significantly alleviated reactive microgliosis and astrogliosis in the corpus callosum, as measured by Iba- 1 and GFAP staining. Moreover, metformin treatment significantly downregulated the expression of pro-inflammatory associated genes (iNOS, H2-Aa, and TNF- $\alpha$ ) in the corpus callosum, whereas expression of anti-inflammatory markers (Arg1, Mrc1, and IL10) was not promoted, compared to CPZ mice . Furthermore, protein levels of iNOS (proinflammatory marker) were significantly decreased in the metformin group, while those of Trem2 (anti-inflammatory marker) were increased. In addition, metformin significantly increased AMPK activation in CPZ mice. Finally, metformin administration significantly reduced the activation level of NF- $\kappa \mathrm{B}$ in $\mathrm{CPZ}$ mice. In summary, our data revealed that metformin attenuated pro-inflammatory microglia markers through suppressing NF- $\mathrm{BB}$ activity. The positive effects of metformin on microglia and remyelination suggest that it could be used as a promising candidate to lessen the incidence of inflammatory neurodegenerative diseases such as MS. @ 2021, The Author(s), under exclusive licence to Springer Science+Business Media, LLC, part of Springer Nature.

\section{Author keywords}

Cuprizone; Metformin ; Microglia activity; Multiple sclerosis; Neuroinflammation; NF- $\mathrm{B}$

\section{Reaxys Chemistry database information (i)}

\{"fill-

opacity":"1","colorrendering":"auto","colorinterpolation":"auto","textrendering":"auto","stroke": Nirecelptạllsquare","width":" miterlimit":"10","shape-

Powered by Reaxys \{"fill-

opacity":"1","colorrendering":"auto","colorinterpolation":"auto","textrendering":"auto","stroke":"black","strokeMiacd ptấlsquare","width":"200","'strokemiterlimit":"10","shape-

SciVal Topics (i)

Metrics

Funding details 
1 Adilijiang, A., Guan, T., He, J., Hartle, K., Wang, W., Li, X.

The protective effects of areca catechu extract on cognition and social interaction deficits in a cuprizone-induced demyelination model (Open Access)

(2015) Evidence-based Complementary and Alternative Medicine, 2015, art. no. 426092 . Cited 20 times.

http://www.hindawi.com/journals/ecam/contents.html

doi: $10.1155 / 2015 / 426092$

View at Publisher

2 Algire, C., Moiseeva, O., Deschênes-Simard, X., Amrein, L., Petruccelli, L., Birman, E., Viollet, B., (...), Pollak, M.N.

Metformin reduces endogenous reactive oxygen species and associated DNA damage

(2012) Cancer Prevention Research, 5 (4), pp. 536-543. Cited 216 times. http://cancerpreventionresearch.aacrjournals.org/content/5/4/536.full.pdf+ht $\underline{\mathrm{ml}}$

doi: 10.1158/1940-6207.CAPR-11-0536

View at Publisher

3 Amende, I., Kale, A., McCue, S., Glazier, S., Morgan, J.P., Hampton, T.G.

Gait dynamics in mouse models of Parkinson's disease and Huntington's disease (Open Access)

(2005) Journal of NeuroEngineering and Rehabilitation, 2. Cited 110 times. http://www.jneuroengrehab.com/content/pdf/1743-0003-2-20.pdf doi: $10.1186 / 1743-0003-2-20$

View at Publisher

4 Arnoux, I., Willam, M., Griesche, N., Krummeich, J., Watari, H., Offermann, N., Weber, S., (...), Stroh, A.

Metformin reverses early cortical network dysfunction and behavior changes in Huntington's disease (Open Access)

(2018) eLife, 7, art. no. e38744. Cited 31 times.

https://elifesciences.org/download/aHR0cHM6Ly9jZG4uZWxpZmVzY2IlbmN Icy5vcmcvYXJ0aWNsZXMvMzg3NDQvZWxpZmUtMzg3NDQtdjMucGRm/eli fe-38744-v3.pdf?

hash=bjkDMnsO\%2BwBP5 pupWE6 p0\%2F2SrPm6wQ0VsVRcff5uaSs\%3D

doi: 10.7554/eLife.38744

View at Publisher

5 Aryanpour, R., Pasbakhsh, P., Zibara, K., Namjoo, Z., Beigi Boroujeni, F., Shahbeigi, S., Kashani, I.R., (...), Zendehdel, A.

Progesterone therapy induces an $M 1$ to $M 2$ switch in microglia phenotype and suppresses NLRP3 inflammasome in a cuprizone-induced demyelination mouse model

(2017) International Immunopharmacology, 51, pp. 131-139. Cited 65 times. www.elsevier.com/locate/intimp doi: 10.1016/j.intimp.2017.08.007

View at Publisher 
6 Aryanpour, R., Pasbakhsh, P., Zibara, K., Namjoo, Z., Beigi Boroujeni, F., Shahbeigi, S., Kashani, I.R., (...), Zendehdel, A.

Progesterone therapy induces an $\mathrm{Ml}$ to $\mathrm{M} 2$ switch in microglia phenotype and suppresses NLRP3 inflammasome in a cuprizone-induced demyelination mouse model

(2017) International Immunopharmacology, 51, pp. 131-139. Cited 65 times. www.elsevier.com/locate/intimp doi: 10.1016/j.intimp.2017.08.007

View at Publisher

7 Aryanpour, R., Zibara, K., Pasbakhsh, P., Jame'ei, S.B., Namjoo, Z., Ghanbari, A., Mahmoudi, R., (...), Kashani, I.R.

$17 \beta$-Estradiol Reduces Demyelination in Cuprizone-fed Mice by Promoting M2 Microglia Polarity and Regulating NLRP3 Inflammasome

(2021) Neuroscience, 463, pp. 116-127. www.elsevier.com/locate/neuroscience doi: 10.1016/j.neuroscience.2021.03.025

View at Publisher

Barati, S., Ragerdi Kashani, I., Moradi, F., Tahmasebi, F., Mehrabi, S., Barati, M., Joghataei, M.T.

Mesenchymal stem cell mediated effects on microglial phenotype in cuprizone-induced demyelination model

(2019) Journal of Cellular Biochemistry, 120 (8), pp. 13952-13964. Cited 15 times.

http://onlinelibrary.wiley.com/journal/10.1002/(ISSN)1097-4644

doi: $10.1002 / j c b .28670$

View at Publisher

9 Bernardes, D., Oliveira, A.L.R.

Comprehensive catwalk gait analysis in a chronic model of multiple sclerosis subjected to treadmill exercise training (Open Access)

(2017) BMC Neurology, 17 (1), art. no. 160. Cited 14 times. http://www.biomedcentral.com/bmcneurol/ doi: 10.1186/s12883-017-0941-z

View at Publisher

10 Bonetti, B., Stegagno, C., Moretto, G., Rizzuto, N., Cannella, B., Raine, C. Localization of NFkB in multiple sclerosis lesions: implications for oligodendrocyte damage

(1998) J Neuroimmunol, 90 (1), p. 71.

11 Brousse, B., Mercier, O., Magalon, K., Daian, F., Durbec, P., Cayre, M. Endogenous neural stem cells modulate microglia and protect against demyelination (Open Access)

(2021) Stem Cell Reports, 16 (7), pp. 1792-1804.

http://www.elsevier.com/journals/stem-cell-reports/2213-6711

doi: 10.1016/j.stemcr.2021.05.002

View at Publisher 
12 Brück, W., Pförtner, R., Pham, T., Zhang, J., Hayardeny, L., Piryatinsky, V., Hanisch, U.-K., (...), Wegner, C.

Reduced astrocytic NF-KB activation by laquinimod protects from cuprizone-induced demyelination (Open Access)

(2012) Acta Neuropathologica, 124 (3), pp. 411-424. Cited 125 times. doi: 10.1007/s00401-012-1009-1

View at Publisher

Cai, Z., Hussain, M.D., Yan, L.-J.

Microglia, neuroinflammation, and beta-amyloid protein in Alzheimer's disease

(2014) International Journal of Neuroscience, 124 (5), pp. 307-321. Cited 262

times.

www.tandf.co.uk/journals/titles/00207454.asp

doi: $10.3109 / 00207454.2013 .833510$

View at Publisher

14 Cantoni, C., Bollman, B., Licastro, D., Xie, M., Mikesell, R., Schmidt, R., Yuede, C.M., (...), Piccio, L.

TREM2 regulates microglial cell activation in response to demyelination in vivo (Open Access)

(2015) Acta Neuropathologica, 129 (3), pp. 429-447. Cited 123 times. link.springer.de/link/service/journals/00401/index.htm doi: 10.1007/s00401-015-1388-1

View at Publisher

15 Cherry, J.D., Olschowka, J.A., O'Banion, M.K.

Neuroinflammation and M2 microglia: The good, the bad, and the inflamed (Open Access)

(2014) Journal of Neuroinflammation, 11, art. no. 98. Cited 827 times. http://www.jneuroinflammation.com/content/11/1/98 doi: 10.1186/1742-2094-11-98

View at Publisher

Chu, F., Shi, M., Zheng, C., Shen, D., Zhu, J., Zheng, X., Cui, L.

The roles of macrophages and microglia in multiple sclerosis and experimental autoimmune encephalomyelitis

(2018) Journal of Neuroimmunology, 318, pp. 1-7. Cited 109 times. www.elsevier.com/locate/jneuroim doi: 10.1016/j.jneuroim.2018.02.015

View at Publisher

17 Cignarella, F., Filipello, F., Bollman, B., Cantoni, C., Locca, A., Mikesell, R., Manis, M., (...), Piccio, L.

TREM2 activation on microglia promotes myelin debris clearance and remyelination in a model of multiple sclerosis (Open Access)

(2020) Acta Neuropathologica, 140 (4), pp. 513-534. Cited 31 times. link.springer.de/link/service/journals/00401/index.htm doi: 10.1007/s00401-020-02193-z

View at Publisher 
18 Citraro, R., Leo, A., Constanti, A., Russo, E., De Sarro, G.

MTOR pathway inhibition as a new therapeutic strategy in epilepsy and epileptogenesis (Open Access)

(2016) Pharmacological Research, 107, pp. 333-343. Cited 106 times. http://www.elsevier.com/inca/publications/store/6/2/2/9/3/1/index.htt doi: 10.1016/j.phrs.2016.03.039

View at Publisher

19 Dendrou, C.A., Fugger, L., Friese, M.A.

Immunopathology of multiple sclerosis

(2015) Nature Reviews Immunology, 15 (9), pp. 545-558. Cited 981 times. http://www.nature.com/nri/index.html

doi: $10.1038 /$ nri3871

View at Publisher

20 Devanney, N.A., Stewart, A.N., Gensel, J.C.

Microglia and macrophage metabolism in CNS injury and disease: The role of immunometabolism in neurodegeneration and neurotrauma (Open Access)

(2020) Experimental Neurology, 329, art. no. 113310. Cited 36 times. http://www.elsevier.com/inca/publications/store/6/2/2/8/2/8/index.htt doi: 10.1016/j.expneurol.2020.113310

View at Publisher

21 Elbaz, E.M., Senousy, M.A., El-Tanbouly, D.M., Sayed, R.H.

Neuroprotective effect of linagliptin against cuprizoneinduced demyelination and behavioural dysfunction in mice: A pivotal role of AMPK/SIRT1 and JAK2/STAT3/NF-KB signalling pathway modulation

(2018) Toxicology and Applied Pharmacology, 352, pp. 153-161. Cited 33 times.

http://www.elsevier.com/inca/publications/store/6/2/2/9/5/1/index.htt doi: 10.1016/j.taap.2018.05.035

View at Publisher

22 Elbaz, E.M., Senousy, M.A., El-Tanbouly, D.M., Sayed, R.H.

Neuroprotective effect of linagliptin against cuprizoneinduced demyelination and behavioural dysfunction in mice: A pivotal role of AMPK/SIRTI and JAK2/STAT3/NF-KB signalling pathway modulation

(2018) Toxicology and Applied Pharmacology, 352, pp. 153-161. Cited 33

times.

http://www.elsevier.com/inca/publications/store/6/2/2/9/5/1/index.htt

doi: 10.1016/j.taap.2018.05.035

View at Publisher 
23 Goldberg, J., Clarner, T., Beyer, C., Kipp, M.

Anatomical Distribution of Cuprizone-Induced Lesions in C57BL6 Mice

(2015) Journal of Molecular Neuroscience, 57 (2), pp. 166-175. Cited 49

times.

http://www.springer.com/humana+press/journal/12031

doi: 10.1007/s12031-015-0595-5

View at Publisher

24 Groebe, A., Clarner, T., Baumgartner, W., Dang, J., Beyer, C., Kipp, M.

Cuprizone treatment induces distinct demyelination, astrocytosis, and microglia cell invasion or proliferation in the mouse cerebellum

(2009) Cerebellum, 8 (3), pp. 163-174. Cited 82 times.

doi: 10.1007/s12311-009-0099-3

View at Publisher

25 Gudi, V., Gingele, S., Skripuletz, T., Stangel, M.

Glial response during cuprizone-induced de- and

remyelination in the CNS: Lessons learned (Open Access)

(2014) Frontiers in Cellular Neuroscience, 8 (MAR), art. no. 73. Cited 183

times.

http://journal.frontiersin.org/Journal/10.3389/fncel.2014.00073/pdf

doi: $10.3389 /$ fncel.2014.00073

View at Publisher

26 Guerrero, B.L., Sicotte, N.L.

Microglia in Multiple Sclerosis: Friend or Foe? (Open Access)

(2020) Frontiers in Immunology, 11, art. no. 374. Cited 32 times.

https://www.frontiersin.org/journals/immunology\#

doi: $10.3389 /$ fimmu.2020.00374

View at Publisher

27 Gveric, D., Kaltschmidt, C., Cuzner, M.L., Newcombe, J.

Transcription factor NF-KB and inhibitor IKBa are localized in macrophages in active multiple sclerosis lesions (Open Access)

(1998) Journal of Neuropathology and Experimental Neurology, 57 (2), pp.

168-178. Cited 94 times.

http://journals.lww.com/jneuropath

doi: 10.1097/00005072-199802000-00008

View at Publisher

28 Hattori, Y., Suzuki, K., Hattori, S., Kasai, K.

Metformin inhibits cytokine-induced nuclear factor $\mathrm{KB}$ activation via AMP-activated protein kinase activation in vascular endothelial cells (Open Access)

(2006) Hypertension, 47 (6), pp. 1183-1188. Cited 343 times.

doi: 10.1161/01.HYP.0000221429.94591.72

View at Publisher 
29 Houshmand, F., Barati, M., Golab, F., Ramezani-sefidar, S., Tanbakooie, S., Tabatabaei, M., Amiri, M., (...), Sanadgol, N.

Metformin-induced AMPK activation stimulates remyelination through induction of neurotrophic factors, downregulation of NogoA and recruitment of Olig2+ precursor cells in the cuprizone murine model of multiple sclerosis (Open Access)

(2019) DARU, Journal of Pharmaceutical Sciences, 27 (2), pp. 583-592. Cited 8

times.

https://rd.springer.com/journal/40199

doi: $10.1007 / s 40199-019-00286-z$

View at Publisher

30 Houshmand, F., Barati, M., Golab, F., Ramezani-sefidar, S., Tanbakooie, S., Tabatabaei, M., Amiri, M., (...), Sanadgol, N.

Metformin-induced AMPK activation stimulates remyelination through induction of neurotrophic factors, downregulation of NogoA and recruitment of Olig2+ precursor cells in the cuprizone murine model of multiple sclerosis (Open Access)

(2019) DARU, Journal of Pharmaceutical Sciences, 27 (2), pp. 583-592. Cited 8 times.

https://rd.springer.com/journal/40199

doi: $10.1007 / s 40199-019-00286-z$

View at Publisher

31 Hwang, I.K., Kim, I.Y., Joo, E.J., Shin, J.H., Choi, J.W., Won, M.-H., Yoon, Y.S., (...), Seong, J.K.

Metformin normalizes type 2 diabetes-induced decrease in cell proliferation and neuroblast differentiation in the rat dentate gyrus

(2010) Neurochemical Research, 35 (4), pp. 645-650. Cited 29 times. doi: 10.1007/s11064-009-0115-5

View at Publisher

32 laccarino, H.F., Singer, A.C., Martorell, A.J., Rudenko, A., Gao, F., Gillingham, T.Z., Mathys, H., (...), Tsai, L.-H.

Gamma frequency entrainment attenuates amyloid load and modifies microglia (Open Access)

(2016) Nature, 540 (7632), pp. 230-235. Cited 395 times.

http://www.nature.com/nature/index.html

doi: $10.1038 /$ nature20587

View at Publisher

33 Jeon, S.-M.

Regulation and function of AMPK in physiology and diseases (Open Access)

(2016) Experimental \& molecular medicine, 48 (7), p. e245. Cited 422 times. doi: $10.1038 / \mathrm{emm} .2016 .81$

View at Publisher 
34 Jha, M.K., Lee, W.-H., Suk, K.

Functional polarization of neuroglia: Implications in neuroinflammation and neurological disorders

(2016) Biochemical Pharmacology, 103, pp. 1-16. Cited 131 times. www.elsevier.com/locate/biochempharm

doi: 10.1016/j.bcp.2015.11.003

View at Publisher

35 Jin, Q., Cheng, J., Liu, Y., Wu, J., Wang, X., Wei, S., Zhou, X., (...), Zhen, X. Improvement of functional recovery by chronic metformin treatment is associated with enhanced alternative activation of microglia/macrophages and increased angiogenesis and neurogenesis following experimental stroke

(2014) Brain, Behavior, and Immunity, 40, pp. 131-142. Cited 173 times. http://www.elsevier.com/inca/publications/store/6/2/2/8/0/0/index.htt doi: 10.1016/j.bbi.2014.03.003

View at Publisher

Jing, Y., Wu, F., Li, D., Yang, L., Li, Q., Li, R.

Metformin improves obesity-associated inflammation by altering macrophages polarization

(2018) Molecular and Cellular Endocrinology, 461, pp. 256-264. Cited 84 times.

www.elsevier.com/locate/mce

doi: 10.1016/j.mce.2017.09.025

View at Publisher

37 Kheirandish, M., Mahboobi, H., Yazdanparast, M., Kamal, W., Kamal, M.A. Anti-cancer effects of metformin: Recent evidences for its role in prevention and treatment of cancer

(2018) Current Drug Metabolism, 19 (9), pp. 793-797. Cited 36 times. http://www.eurekaselect.com/161323

doi: $10.2174 / 1389200219666180416161846$

View at Publisher

38 Kim, J., Kundu, M., Viollet, B., Guan, K.L.

AMPK and $\mathrm{mTOR}$ regulate autophagy through direct phosphorylation of Ulkl (Open Access)

(2011) Nature Cell Biology, 13 (2), pp. 132-141. Cited 3804 times. doi: $10.1038 /$ ncb2152

View at Publisher

39 Kim, J., Yang, G., Kim, Y., Kim, J., Ha, J.

AMPK activators: Mechanisms of action and physiological activities (Open Access)

(2016) Experimental and Molecular Medicine, 48 (4), art. no. e224. Cited 295

times.

http://www.nature.com/press_releases/emm-relaunch.html

doi: $10.1038 / \mathrm{emm} .2016 .16$

View at Publisher 
40 Kim, W., Hahn, K.R., Jung, H.Y., Kwon, H.J., Nam, S.M., Kim, J.W., Park, J.H., (...), Hwang, I.K.

Melatonin ameliorates cuprizone-induced reduction of hippocampal neurogenesis, brain-derived neurotrophic factor, and phosphorylation of cyclic AMP response element-binding protein in the mouse dentate gyrus (Open Access)

(2019) Brain and Behavior, 9 (9), art. no. e01388. Cited 14 times. http://onlinelibrary.wiley.com/journal/10.1002/(ISSN)2157-9032 doi: $10.1002 /$ brb3.1388

View at Publisher

41 Kipp, M., Clarner, T., Dang, J., Copray, S., Beyer, C.

The cuprizone animal model: New insights into an old story

(2009) Acta Neuropathologica, 118 (6), pp. 723-736. Cited 309 times. doi: 10.1007/s00401-009-0591-3

View at Publisher

42 Kiriyama, Y., Nochi, $\mathrm{H}$.

The function of autophagy in neurodegenerative diseases

(Open Access)

(2015) International Journal of Molecular Sciences, 16 (11), pp. 26797-

26812. Cited 99 times.

http://www.mdpi.com/1422-0067/16/11/25990/pdf

doi: $10.3390 /$ ijms 161125990

View at Publisher

43 Kosaraju, J., Seegobin, M., Gouveia, A., Syal, C., Sarma, S.N., Lu, K.J., Ilin, J., (...), Wang, J.

Metformin promotes CNS remyelination and improves social interaction following focal demyelination through CBP Ser436 phosphorylation

(2020) Experimental Neurology, 334, art. no. 113454.

http://www.elsevier.com/inca/publications/store/6/2/2/8/2/8/index.htt doi: 10.1016/j.expneurol.2020.113454

View at Publisher

44 Largani, S.H.H., Borhani-Haghighi, M., Pasbakhsh, P., Mahabadi, V.P., Nekoonam, S., Shiri, E., Kashani, I.R., (...), Zendehdel, A.

Oligoprotective effect of metformin through the AMPKdependent on restoration of mitochondrial hemostasis in the cuprizone-induced multiple sclerosis model

(2019) Journal of Molecular Histology, 50 (3), pp. 263-271. Cited 11 times. http://www.kluweronline.com/issn/1567-2379/

doi: $10.1007 /$ s10735-019-09824-0

View at Publisher 
45 Lassmann, $\mathrm{H}$.

Multiple sclerosis pathology (Open Access)

(2018) Cold Spring Harbor Perspectives in Medicine, 8 (3), art. no. a028936. Cited 183 times.

http://perspectivesinmedicine.cshlp.org/content/8/3/a028936.full.pdf

doi: $10.1101 /$ cshperspect.a028936

View at Publisher

6 Lassmann, H., Van Horssen, J., Mahad, D.

Progressive multiple sclerosis: Pathology and pathogenesis

(2012) Nature Reviews Neurology, 8 (11), pp. 647-656. Cited 556 times. http://www.nature.com/nrneurol/archive/index.html

doi: $10.1038 /$ nrneurol.2012.168

View at Publisher

47

Legro, R.S., Barnhart, H.X., Schlaff, W.D., Carr, B.R., Diamond, M.P., Carson, S.A., Steinkampf, M.P., (...), Myers, E.R.

Clomiphene, metformin, or both for infertility in the polycystic ovary syndrome (Open Access)

(2007) New England Journal of Medicine, 356 (6), pp. 551-566. Cited 736

times.

http://content.nejm.org/cgi/reprint/356/6/551.pdf

doi: 10.1056/NEJMoa063971

View at Publisher

$48 \mathrm{Li}$, J., Deng, J., Sheng, W., Zuo, Z.

Metformin attenuates Alzheimer's disease-like neuropathology in obese, leptin-resistant mice (Open Access)

(2012) Pharmacology Biochemistry and Behavior, 101 (4), pp. 564-574. Cited 168 times.

doi: 10.1016/j.pbb.2012.03.002

View at Publisher

Lu, M., Su, C., Qiao, C., Bian, Y., Ding, J., Hu, G.

Metformin prevents dopaminergic neuron death in MPTP/Pinduced mouse model of Parkinson's disease via autophagy and mitochondrial ROS clearance (Open Access)

(2016) International Journal of Neuropsychopharmacology, 19 (9), pp. 1-

11. Cited 117 times.

http://ijnp.oxfordjournals.org/

doi: 10.1093/ijnp/pyw047

View at Publisher

0 Luo, C., Jian, C., Liao, Y., Huang, Q., Wu, Y., Liu, X., Zou, D., (...), Wu, Y.

The role of microglia in multiple sclerosis (Open Access)

(2017) Neuropsychiatric Disease and Treatment, 13, pp. 1661-1667. Cited 74 times.

https://www.dovepress.com/getfile.php?filelD=37107 doi: $10.2147 /$ NDT.S140634

View at Publisher 
51 Manwani, B., Mccullough, L.D.

Function of the master energy regulator adenosine monophosphate-activated protein kinase in stroke (Open Access)

(2013) Journal of Neuroscience Research, 91 (8), pp. 1018-1029. Cited 54

times.

doi: 10.1002/jnr.23207

View at Publisher

52 Mc Guire, C., Prinz, M., Beyaert, R., van Loo, G.

Nuclear factor kappa B (NF-KB) in multiple sclerosis pathology

(2013) Trends in Molecular Medicine, 19 (10), pp. 604-613. Cited 88 times. doi: 10.1016/j.molmed.2013.08.001

View at Publisher

53 Memmott, R.M., Mercado, J.R., Maier, C.R., Kawabata, S., Fox, S.D., Dennis, P.A.

Metformin prevents tobacco carcinogen-induced lung tumorigenesis (Open Access)

(2010) Cancer Prevention Research, 3 (9), pp. 1066-1076. Cited 323 times. http://cancerpreventionresearch.aacrjournals.org/content/3/9/1066.full.pdf+ht $\underline{\mathrm{ml}}$

doi: 10.1158/1940-6207.CAPR-10-0055

View at Publisher

54 Nakagawa, Y., Chiba, K.

Diversity and plasticity of microglial cells in psychiatric and neurological disorders

(2015) Pharmacology and Therapeutics, 154, pp. 21-35. Cited 86 times. www.elsevier.com/locate/pharmthera doi: 10.1016/j.pharmthera.2015.06.010

View at Publisher

55 Nakatake, R., lida, H., Ishizaki, M., Matsui, K., Nakamura, Y., Kaibori, M., Nishizawa, M., (...), Okumura, T.

Metformin inhibits expression of the proinflammatory biomarker inducible nitric oxide synthase in hepatocytes (Open Access)

(2018) Functional Foods in Health and Disease, 8 (3), pp. 175-192. Cited 5 times.

https://ffhdj.com/index.php/ffhd/article/view/423

doi: $10.31989 /$ ffhd.v8i3.423

View at Publisher

56 Nath, N., Khan, M., Paintlia, M.K., Hoda, Md.N., Giri, S.

Metformin attenuated the autoimmune disease of the central nervous system in animal models of multiple sclerosis (Open Access)

(2009) Journal of Immunology, 182 (12), pp. 8005-8014. Cited 236 times. http://www.jimmunol.org/cgi/reprint/182/12/8005 doi: $10.4049 /$ jimmunol.0803563

View at Publisher 
57 Nath, N., Khan, M., Paintlia, M.K., Hoda, Md.N., Giri, S.

Metformin attenuated the autoimmune disease of the central nervous system in animal models of multiple sclerosis

(Open Access)

(2009) Journal of Immunology, 182 (12), pp. 8005-8014. Cited 236 times.

http://www.jimmunol.org/cgi/reprint/182/12/8005

doi: $10.4049 /$ jimmunol.0803563

View at Publisher

58 Nath, N., Khan, M., Rattan, R., Mangalam, A., Makkar, R.S., Meester, C.d., Bertrand, L., (...), Giri, S.

Loss of AMPK exacerbates experimental autoimmune encephalomyelitis disease severity (Open Access)

(2009) Biochemical and Biophysical Research Communications, 386 (1), pp. 16-20. Cited 55 times.

doi: 10.1016/j.bbrc.2009.05.106

View at Publisher

59 Negrotto, L., Farez, M.F., Correale, J.

Immunologic effects of metformin and pioglitazone treatment on metabolic syndrome and multiple sclerosis (Open Access)

(2016) JAMA Neurology, 73 (5), pp. 520-528. Cited 80 times.

http://archneur.jamanetwork.com/data/Journals/NEUR/935258/noil50104.pd f

doi: 10.1001/jamaneurol.2015.4807

View at Publisher

60 Neumann, H., Takahashi, K.

Essential role of the microglial triggering receptor expressed on myeloid cells-2 (TREM2) for central nervous tissue immune homeostasis

(2007) Journal of Neuroimmunology, 184 (1-2), pp. 92-99. Cited 183 times. doi: 10.1016/j.jneuroim.2006.11.032

View at Publisher

61 Nyamoya, S., Leopold, P., Becker, B., Beyer, C., Hustadt, F., Schmitz, C., Michel, A., (...), Kipp, M.

G-Protein-Coupled Receptor Gpr17 Expression in Two Multiple Sclerosis Remyelination Models

(2019) Molecular Neurobiology, 56 (2), pp. 1109-1123. Cited 15 times. http://www.springer.com/biomed/neuroscience/journal/12035 doi: 10.1007/s12035-018-1146-1

View at Publisher

62 Orihuela, R., McPherson, C.A., Harry, G.J.

Microglial M1/M2 polarization and metabolic states (Open Access)

(2016) British Journal of Pharmacology, 173 (4), pp. 649-665. Cited 630 times. http://onlinelibrary.wiley.com/journal/10.1111/\%28ISSN\%291476-5381 doi: 10.1111/bph.13139

View at Publisher 
63 Paintlia, A.S., Paintlia, M.K., Mohan, S., Singh, A.K., Singh, I.

AMP-activated protein kinase signaling protects oligodendrocytes that restore central nervous system functions in an experimental autoimmune encephalomyelitis model (Open Access)

(2013) American Journal of Pathology, 183 (2), pp. 526-541. Cited 36 times. doi: 10.1016/j.ajpath.2013.04.030

View at Publisher

Peixoto, C.A., Oliveira, W.H.D., Araújo, S.M.D.R., Nunes, A.K.S.

AMPK activation: Role in the signaling pathways of neuroinflammation and neurodegeneration

(2017) Experimental Neurology, Part A 298, pp. 31-41. Cited 75 times. http://www.elsevier.com/inca/publications/store/6/2/2/8/2/8/index.htt doi: 10.1016/j.expneurol.2017.08.013

View at Publisher

65 Pott, F., Gingele, S., Clarner, T., Dang, J., Baumgartner, W., Beyer, C., Kipp, M.

Cuprizone effect on myelination, astrogliosis and microglia attraction in the mouse basal ganglia

(2009) Brain Research, 1305, pp. 137-149. Cited 60 times.

www.elsevier.com/locate/bri

doi: 10.1016/j.brainres.2009.09.084

View at Publisher

66 Praet, J., Guglielmetti, C., Berneman, Z., Van der Linden, A., Ponsaerts, P.

Cellular and molecular neuropathology of the cuprizone mouse model: Clinical relevance for multiple sclerosis

(Open Access)

(2014) Neuroscience and Biobehavioral Reviews, 47, pp. 485-505. Cited 203 times.

www.elsevier.com/locate/neubiorev

doi: 10.1016/j.neubiorev.2014.10.004

View at Publisher

Qing, L., Fu, J., Wu, P., Zhou, Z., Yu, F., Tang, J.

Metformin induces the $\mathrm{m} 2$ macrophage polarization to accelerate the wound healing via regulating ampk/mtor/nlrp3 inflammasome singling pathway

(2019) American Journal of Translational Research, 11 (2), art. no. AJTR0085206, pp. 655-668. Cited 55 times. http://www.ajtr.org/files/ajtr0085206.pdf

Saijo, K., Glass, C.K.

Microglial cell origin and phenotypes in health and disease

(2011) Nature Reviews Immunology, 11 (11), pp. 775-787. Cited 675 times. doi: $10.1038 /$ nri3086

View at Publisher 
69 Salminen, A., Hyttinen, J.M.T., Kaarniranta, K.

AMP-activated protein kinase inhibits NF-KB signaling and inflammation: Impact on healthspan and lifespan (Open Access)

(2011) Journal of Molecular Medicine, 89 (7), pp. 667-676. Cited 509 times. doi: 10.1007/s00109-011-0748-0

View at Publisher

70 Sanadgol, N., Barati, M., Houshmand, F., Hassani, S., Clarner, T., shahlaei, M., Golab, F.

Metformin accelerates myelin recovery and ameliorates behavioral deficits in the animal model of multiple sclerosis via adjustment of AMPK/Nrf2/mTOR signaling and maintenance of endogenous oligodendrogenesis during brain self-repairing period

(2020) Pharmacological Reports, 72 (3), pp. 641-658. Cited 7 times.

https://link.springer.com/journal/43440

doi: 10.1007/s43440-019-00019-8

View at Publisher

71 Sanadgol, N., Barati, M., Houshmand, F., Hassani, S., Clarner, T., shahlaei, M., Golab, F.

Metformin accelerates myelin recovery and ameliorates behavioral deficits in the animal model of multiple sclerosis via adjustment of AMPK/Nrf2/mTOR signaling and maintenance of endogenous oligodendrogenesis during brain self-repairing period

(2020) Pharmacological Reports, 72 (3), pp. 641-658. Cited 7 times. https://link.springer.com/journal/43440

doi: $10.1007 / \mathrm{s} 43440-019-00019-8$

View at Publisher

72 Sanadgol, N., Golab, F., Mostafaie, A., Mehdizadeh, M., Khalseh, R., Mahmoudi, M., Abdollahi, M., (...), Sharifzadeh, M.

Low, but not high, dose triptolide controls neuroinflammation and improves behavioral deficits in toxic model of multiple sclerosis by dampening of NF-KB activation and acceleration of intrinsic myelin repair

(2018) Toxicology and Applied Pharmacology, 342, pp. 86-98. Cited 17 times. http://www.elsevier.com/inca/publications/store/6/2/2/9/5/1/index.htt doi: 10.1016/j.taap.2018.01.023

View at Publisher

73 Sanchez-Guajardo, V., Tentillier, N., Romero-Ramos, M.

The relation between $\alpha$-synuclein and microglia in Parkinson's disease: Recent developments

(2015) Neuroscience, 302, pp. 47-58. Cited 104 times.

www.elsevier.com/locate/neuroscience

doi: 10.1016/j.neuroscience.2015.02.008

View at Publisher 
74 Srinivasan, M., Lahiri, D.K.

Significance of NF-KB as a pivotal therapeutic target in the neurodegenerative pathologies of Alzheimer's disease and multiple sclerosis (Open Access)

(2015) Expert Opinion on Therapeutic Targets, 19 (4), pp. 471-487. Cited 95 times.

doi: $10.1517 / 14728222.2014 .989834$

View at Publisher

75 Sun, J., Huang, N., Ma, W., Zhou, H., Lai, K.

Protective effects of metformin on lipopolysaccharide-induced airway epithelial cell injury via NF-KB signaling inhibition

(Open Access)

(2019) Molecular Medicine Reports, 19 (3), pp. 1817-1823. Cited 14 times. https://www.spandidos-publications.com/mmr/19/3/1809/download doi: $10.3892 / \mathrm{mmr} .2019 .9807$

View at Publisher

Sun, Y., Tian, T., Gao, J., Liu, X., Hou, H., Cao, R., Li, B., (...), Guo, L.

Metformin ameliorates the development of experimental autoimmune encephalomyelitis by regulating Thelper 17 and regulatory $T$ cells in mice

(2016) Journal of Neuroimmunology, 292, pp. 58-67. Cited 64 times. www.elsevier.com/locate/jneuroim doi: 10.1016/j.jneuroim.2016.01.014

View at Publisher

77 Tang, Y., Le, W.

Differential Roles of $\mathrm{Ml}$ and M2 Microglia in Neurodegenerative Diseases

(2016) Molecular Neurobiology, 53 (2), pp. 1181-1194. Cited 779 times. http://www.springer.com/biomed/neuroscience/journal/12035 doi: 10.1007/s12035-014-9070-5

View at Publisher

78 Tayara, K., Espinosa-Oliva, A.M., García-Domínguez, I., Ismaiel, A.A., BozaSerrano, A., Deierborg, T., Machado, A., (...), de Pablos, R.M.

Divergent effects of metformin on an inflammatory model of Parkinson's disease (Open Access)

(2018) Frontiers in Cellular Neuroscience, 12, art. no. 440. Cited 12 times. https://www.frontiersin.org/articles/10.3389/fncel.2018.00440/pdf doi: $10.3389 /$ fncel.2018.00440

View at Publisher

Torkildsen, Ø., Brunborg, L.A., Myhr, K.-M., Bø, L.

The cuprizone model for demyelination

(2008) Acta Neurologica Scandinavica, 117 (SUPPL. 188), pp. 72-76. Cited 170 times.

doi: $10.1111 / j .1600-0404.2008 .01036 . x$

View at Publisher 
80 Vega-Riquer, J.M., Mendez-Victoriano, G., Morales-Luckie, R.A., GonzalezPerez, O.

Five decades of cuprizone, an updated model to replicate demyelinating diseases (Open Access)

(2019) Current Neuropharmacology, 17 (2), pp. 129-141. Cited 45 times. http://www.eurekaselect.com/node/154244 doi: $10.2174 / 1570159 X 15666170717120343$

View at Publisher

$\bigcirc$ Pasbakhsh, P.; Department of Anatomy, school of medicine, Tehran University of Medical Sciences, Tehran, Iran; email:pasbakhs@sina.tums.ac.ir

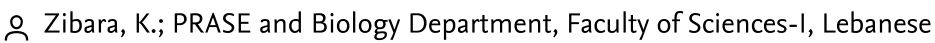

University, Beirut, Lebanon; email:kzibara@ul.edu.lb

(c) Copyright 2021 Elsevier B.V., All rights reserved.

\section{About Scopus}

What is Scopus

Content coverage

Scopus blog

Scopus API

Privacy matters

\section{Language}

日本語に切り替える

切换到简体中文

切換到繁體中文

Русский язык

\section{Customer Service}

Help

Contact us

\section{ELSEVIER}

Terms and conditions $\pi \quad$ Privacy policy $\pi$

Copyright (C) Elsevier B.V ז. All rights reserved. Scopus ${ }^{\circledR}$ is a registered trademark of Elsevier B.V.

We use cookies to help provide and enhance our service and tailor content. By continuing, you agree to the

Q RELX use of cookies. 